\title{
Effect of Corwin (ICI 118587) on resting and exercise heart rate and exercise tolerance in digitalised patients with chronic atrial fibrillation
}

\author{
ADENIYI O MOLAJO, MICHAEL O COUPE, DAVID H BENNETT \\ From the Regional Cardiac Centre, Wythenshawe Hospital, Manchester
}

SUMMARY The effect of Corwin, a new oral beta ${ }_{1}$ partial agonist, on the ventricular response to atrial fibrillation was studied in digitalised patients during 24 hour ambulatory electrocardiography and during exercise on a treadmill in a double blind placebo controlled crossover trial. Corwin reduced the maximum heart rate during exercise from $162(16)$ beats $/ \mathrm{min}$ to $120(9)$ beats/min and reduced the peak heart rate during ambulatory electrocardiography from 113(11) to $90(6)$ beats/min consistent with a beta adrenoreceptor antagonist action at higher levels of sympathetic nervous system activity. Minimum heart rate during ambulatory electrocardiography was increased from 62(5) to $70(5)$ beats/min indicating that at lower levels of sympathetic activity the drug acts as a beta agonist. The drug increased exercise tolerance significantly. Serum digoxin concentrations were not affected by the drug.

Thus Corwin appears to be effective in stabilising heart rate during atrial fibrillation both at rest and during exercise in digitalised patients.

It has been shown that digoxin may be inadequate in controlling the ventricular response to atrial fibrillation during both exercise and stress in some patients. ${ }^{2}$

Corwin $(( \pm)$ - 1 - (4 - hydroxyphenoxy) - 3 - [2 - (4 morpholine carbonamido) ethylamino] - propan - 2 ol fumerate) is a new beta ${ }_{1}$ adrenoceptor partial agonist, which is currently being evaluated for the treatment of mild to moderate cardiac failure and angina. Animal experiments have shown that it attenuates the chronotropic response to sympathetic stimulation. ${ }^{3}$ In normal volunteers ${ }^{4}$ and in a single blind placebo controlled study in 10 patients with moderately severe left ventricular dysfunction, the resting heart rate was unaffected but the chronotropic response to higher levels of exercise was attenuated by the drug (unpublished observations). Observations in normal volunteers have shown an increase in systolic blood pressure and shortening of systolic time intervals consistent with positive inotropic properties.

The purpose of this study was $(a)$ to determine the

Requests for reprints to Dr A O Molajo, Regional Cardiac Centre, Wythenshawe Hospital, Southmoor Road, Wythenshawe, Manchester M23 9LT.

Accepted for publication 26 June 1984 efficacy of oral Corwin in controlling heart rate over a 24 hour period during chronic atrial fibrillation, $(b)$ to evaluate its efficacy in controlling heart rate response during maximal exercise in patients with chronic atrial fibrillation, $(c)$ to study its effect on exercise tolerance in patients with chronic atrial fibrillation, and $(d)$ to study its effect on serum digoxin concentrations in patients with chronic atrial fibrillation.

\section{Patients and methods}

\section{STUDY POPULATION}

Ten patients (eight women and two men, mean age $52(8.7)$ years, range $39-69$ years) were studied. Table 1 gives the clinical diagnosis and treatment. Three patients had symptoms in the New York Heart Association (NYHA) class I, five in class II, and two in class III. They all had documented atrial fibrillation of more than one year's duration. None took any other antiarrhythmic agent apart from digoxin. For admission to the study, the serum digoxin concentration 10 hours after the last dose had to be $\geqslant 0.7 \mathrm{ng} /$ ml. ${ }^{15}$

Informed consent to participate in the study was obtained from all patients. 
Table 1 Clinical data

\begin{tabular}{rllll}
\hline Case No & $\begin{array}{l}\text { Age (yr) } \\
\text { and sex }\end{array}$ & Heart disease & $\begin{array}{l}\text { Functional class } \\
\text { (NYHA) }\end{array}$ & $\begin{array}{l}\text { Digoxin } \\
\text { (mg/day) }\end{array}$ \\
\hline 1 & $69 \mathrm{~F}$ & RHD (MS) & III & 0.25 \\
2 & $60 \mathrm{~F}$ & RHD (MS) & I & 0.25 \\
3 & $60 \mathrm{~F}$ & RHD (MS), mitral & II & 0.25 \\
4 & commissurotomy & III & 0.25 \\
5 & $64 \mathrm{~F}$ & RHD, MVR & II & 0.25 \\
6 & $53 \mathrm{M}$ & Lone AF & II & 0.375 \\
7 & $55 \mathrm{M}$ & IHD & II & 0.25 \\
8 & $48 \mathrm{~F}$ & RHD (MR, MS) & 0.25 \\
9 & $42 \mathrm{~F}$ & RHD (MS), mitral & I & 0.375 \\
10 & $39 \mathrm{~F}$ & RHD (MS and MR) & II & 0.25 \\
\hline
\end{tabular}

RHD, rheumatic heart disease; MR, mitral regurgitation; MS, mitral stenosis; lone AF, atrial fibrillation of unknown aetiology; IHD, ischaemic heart disease; MVR, mitral valve replacement; NYHA, New York Heart Association classification.

\section{STUDY PROCEDURE}

A double blind placebo controlled crossover study was carried out, in which Corwin and digoxin and placebo and digoxin were given alternately for two weeks. There was a one week washout period with placebo and digoxin between the treatment phases. All patients continued to take their usual doses of digoxin and diuretics. Digoxin was given at night so that exercise tests could be performed in the morning at least $\mathbf{1 0}$ hours after the last dose. Depending on the randomisation, they also took Corwin $200 \mathrm{mg}$ or placebo orally twice daily.

A 24 hour ambulatory electrocardiogram was recorded and exercise tolerance and serum digoxin concentrations determined at the start of the study and at the end of each treatment phase. Exercise tolerance was measured on the Marquette TM case computer assisted system for exercise (Marquette Electronics Inc, Milwaukee, USA) using the standard Bruce protocol. ${ }^{6} \mathrm{~A}$ maximal symptom limited exercise test was performed. Modified leads V1, V5, and aVF were continuously monitored during the test. Serum digoxin concentrations were determined at each exercise test 10-12 hours after the last dose of digoxin. The double product was derived from the product of the systolic blood pressure and heart rate at the end of each stage of exercise.

All results are given as mean (standard deviation) and analysed using the paired $t$ test.

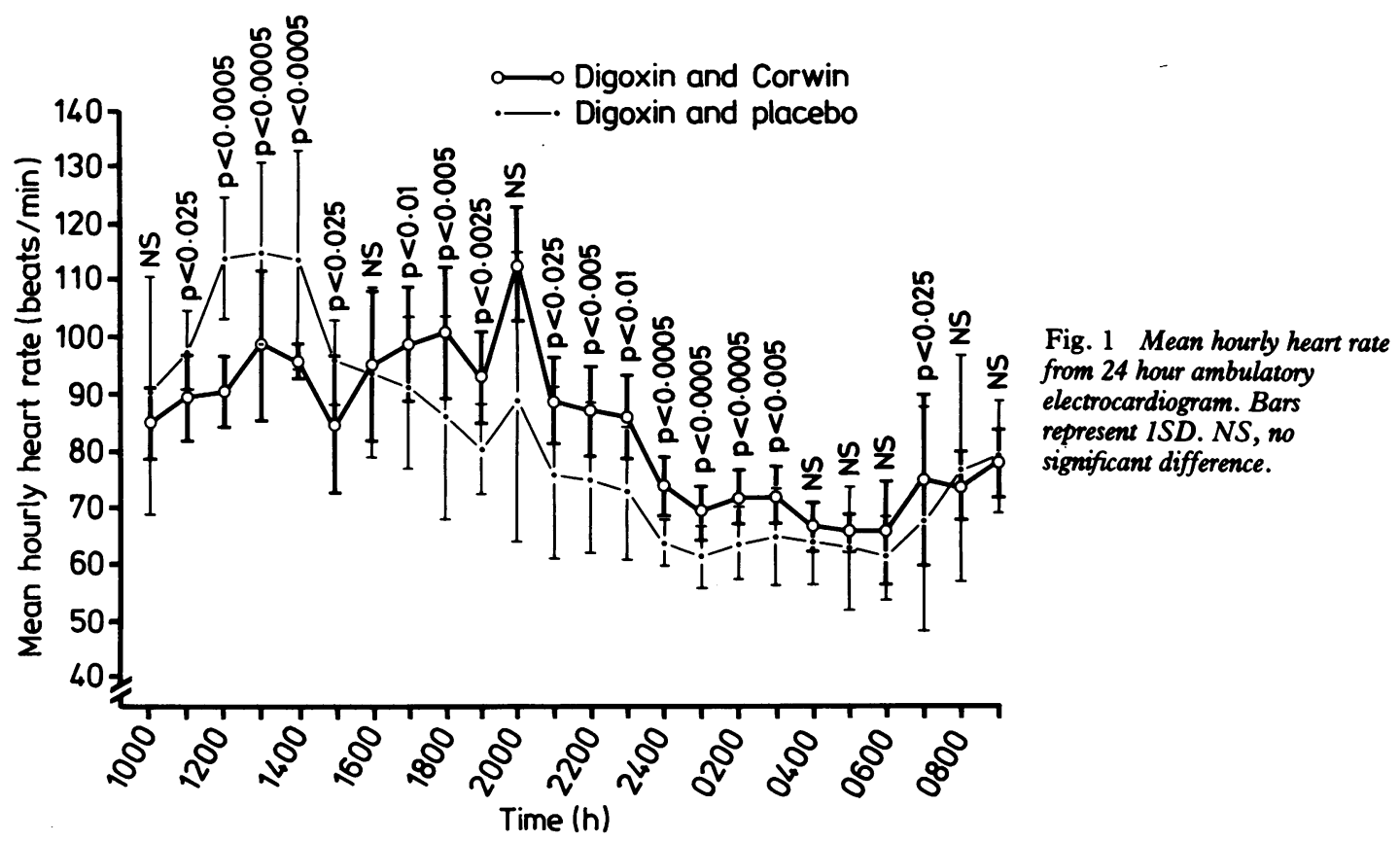


Table 2 Effect of Corwin and placebo on exercise testing and serum digoxin concentrations. Values are mean (SD)

\begin{tabular}{lll}
\hline & Treatment regimen & \\
\cline { 2 - 3 } & $\begin{array}{l}\text { Corwin and } \\
\text { digoxin }\end{array}$ & $\begin{array}{l}\text { Placebo and } \\
\text { digoxin }\end{array}$ \\
\hline Exercise heart rate (beats/min) & & \\
At rest & $73(10)$ & $80(8)$ \\
At 3 minutes' exercise & $105(7)$ & $132(10)$ \\
At peak exercise & $120(9)$ & $162(16)$ \\
Blood pressure (mmHg) & $130(11)$ & $125(11)$ \\
At rest & $138(11)$ & $146(16)$ \\
At 3 minutes' exercise & $140(9)$ & $157(16)$ \\
At peak exercise & $257(114)$ & $215(111)$ \\
Maximum exercise time (s) & $17024(2063)$ & $24262(3883)$ \\
Double product at peak exercise & $1.3(0.1)$ & $<0.0005$ \\
Serum digoxin concentration $(\mathrm{ng} / \mathrm{ml})$ & $<0.05$ & $<0.0025$ \\
\hline
\end{tabular}

\section{Results}

AMBULATORY ELECTROCARDIOGRAPHY

The mean hourly heart rate during placebo treatment was higher between 0800 hours and 1500 hours than during treatment with Corwin. It was lower with placebo b.tween 1600 hours and 0700 hours (Fig. 1).

\section{EXERCISE TESTING}

Heart rate-The mean resting heart rate with digoxin and placebo was significantly higher than the mean resting heart rate with Corwin $(\mathrm{p}<0.01)$ (Table 2, Fig. 2). After three minutes' exercise the mean heart rate with placebo was significantly higher than that with Corwin $(p<0.0005)$. The peak exercise heart rate was lower with Corwin than with placebo $(\mathrm{p}<0.0005)$.

Blood pressure - There was no significant difference in the resting systemic systolic blood pressure with placebo or Corwin. At three minutes' exercise and at peak exercise the mean systemic systolic blood pressure with placebo was significantly higher than that with Corwin ( $\mathrm{p}<0.05$ and $\mathrm{p}<0.0025$ respectively).

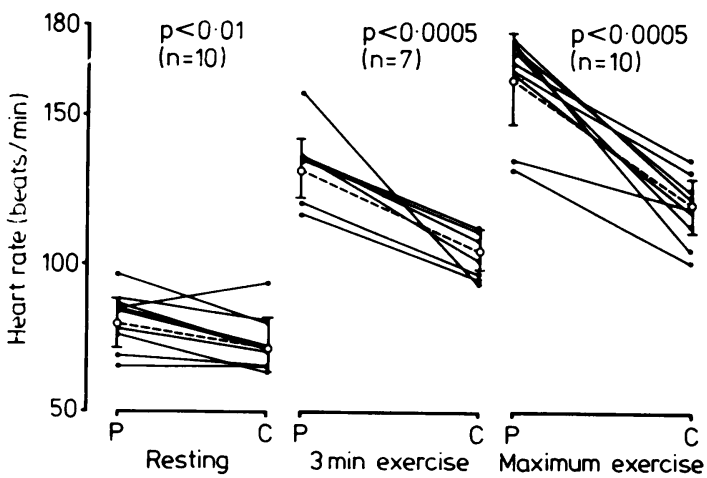

Fig. 2 Effect of Corwin $(C)$ and digoxin and placebo $(P)$ and digoxin on chronotropic response to exercise. individual data; $\bigcirc--\bigcirc$ mean values for the two forms of treatment. Bars represent $1 S D$.
Double product-At peak exercise the mean double product with Corwin was significantly lower than that with placebo $(\mathrm{p}<0.0005)$.

Exercise capacity-The mean maximum exercise time with Corwin was significantly higher than that with placebo $(\mathrm{p}<0.0025)$ (Fig. 3). The three patients with symptoms in NYHA class I showed no significant change in exercise capacity with the two treatments.

\section{SERUM DIGOXIN CONCENTRATIONS AND}

SYMPTOMS

Serum digoxin concentrations with Corwin and placebo were not significantly different. Eight patients reported symptomatic improvement during treatment with Corwin, and two reported no change. None reported any worsening of symptoms.

\section{Discussion}

Corwin is a new beta ${ }_{1}$ adrenoreceptor partial agonist. Previous studies have shown that at rest, when the sympathetic activity is low, it increases the heart rate, whereas during exercise it attenuates the chronotropic response. ${ }^{3}$ This indicates that the beta ${ }_{1}$ antagonist effect of Corwin is evident only when the sympathetic tone is high. In a single blind placebo controlled study in 10 patients with moderately severe left ventricular dysfunction exercise tolerance was improved by the drug (unpublished observations).

In this study heart rate was significantly lower during treatment with Corwin and digoxin than with placebo and digoxin between 1100 hours and 1500 hours. These were periods when the patients were likely to be more active and have a high sympathetic activity; hence Corwin showed its beta ${ }_{1}$ antagonist property. During the period between 1600 and 0300 hours, a period of presumed lesser activity, the hourly heart rate was higher with digoxin and Corwin than with digoxin and placebo. During this period, Corwin probably showed its beta ${ }_{1}$ agonist property.

Although the systemic systolic blood pressure was 


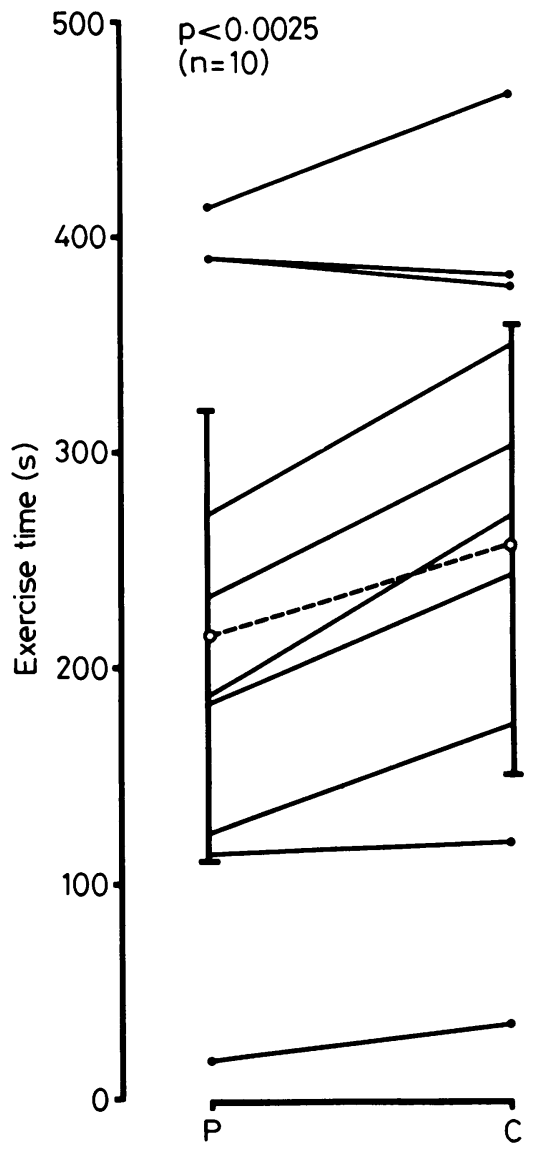

Fig. 3 Effect of Corwin (C) and digoxin and placebo ( $P$ ) and digoxin on maximal exercise time. individual data; $\bigcirc-\ldots$ mean values for the two forms of treatment. Bars represent $1 S D$.

significantly lower with Corwin and digoxin than with digoxin and placebo $(p<0.0025)$, there were no symptoms attributable to this decrease in blood pressure. The double product, an index of myocardial oxygen consumption, was significantly lower with Corwin and digoxin, whereas Rousseau et $\mathrm{al}^{7}$ found neither resting myocardial oxygen consumption nor lactate extraction to be modified by the drug in patients with previous myocardial infarction.

The exercise capacity of the seven patients with impaired exercise tolerance was significantly improved with Corwin and digoxin. During exercise when the sympathetic activity is high, Corwin shows its beta antagonist property: the peak exercise heart rate was significantly lower with Corwin and digoxin than with digoxin and placebo.
In the previous studies showing the beta adrenoreceptor partial agonist activity of Corwin the subjects were in sinus rhythm. The attenuation of the exercise heart rate response in these subjects was probably mediated by the effect of Corwin on the sinus node. By contrast, in this study of patients with atrial fibrillation, the effect of Corwin on exercise heart rate response was presumably mediated by attenuation of the sympathetic enhancement of atrioventricular node conduction.

Unlike verapamil, ${ }^{8}$ whicis may also be used with digoxin to control the ventricular response to atrial fibrillation, Corwin did not affect the serum concentration of digoxin and is thus less likely to lead to digoxin toxicity.

At therapeutic serum concentrations digoxin may be inadequate for the control of ventricular response to atrial fibrillation. ${ }^{12}$ The addition of Corwin orally $200 \mathrm{mg}$ twice daily may lead to a better control of the ventricular response throughout 24 hours. The exercise heart rate response is better controlled with this combination. The serum digoxin concentration is not altered by this addition, and hence the improvement in control is due to the effect of Corwin rather than to an increase in the serum digoxin concentration.

\section{References}

1 Goldman S, Probst P, Selzer A, Cohn K. Inefficacy of "therapeutic" serum levels of digoxin in controlling the ventricular rate in atrial fibrillation. Am $\mathcal{F}$ Cardiol 1975; 35: 651-5.

2 Belic N, Kramer N, Alisuag A, Singer DH, Okita G, Atkinson A. Digoxin: how effective for heart rate control in ambulatory patients with atrial fibrillation? [Abstract]. Circulation 1972; 46 (suppl II): 114.

3 Nuttall A, Snow HM. The cardiovascular effects of ICI

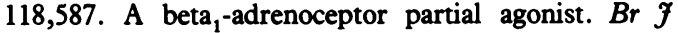
Pharmacol 1982; 77: 381-8.

4 Harry JD, Marlow HF, Wardleworth AG, Young J. The action of ICI 118587 (a beta adrenoreceptor partial agonist) on the heart rate response to exercise in man. $B r f$ Clin Pharmacol 1981; 12: 266P-7P.

5 Smith TW, Butler VP Jr, Haber E. Determination of therapeutic and toxic serum digoxin concentrations by radioimmunoassay. $N$ Engl f Med 1975; 281: 1212-6.

6 Bruce RA, Hornsten TR. Exercise stress testing in evaluation of patients with ischemic heart disease. Prog Cardiovasc Dis 1969; 11: 371-90.

7 Rousseau MF, Pouleur H, Vincent M. Effects of a cardioselective beta ${ }_{1}$ partial agonist (Corwin) on left ventricular function and myocardial metabolism in patients with previous myocardial infarction. Am F Cardiol 1983; 51: 1267-74.

8 Stern EH, Pitchon R, King BD, Guerrero J, Scheider RR, Wiener I. Clinical use of oral verapamil in chronic and paroxysmal atrial fibrillation. Chest 1982; 81: 30811 . 